\title{
Urgences
}

\section{Non à titrer pour introduire l'indéfini dans la déclagration}

\section{André Gervais}

Numéro 21, novembre 1988

Bagatelles et crases

URI : https://id.erudit.org/iderudit/025494ar

DOI : https://doi.org/10.7202/025494ar

Aller au sommaire du numéro

Éditeur(s)

Urgences

ISSN

0226-9554 (imprimé)

1927-3924 (numérique)

Découvrir la revue

Citer ce document

Gervais, A. (1988). Non à titrer pour introduire l'indéfini dans la déclagration. Urgences, (21), 65-76. https://doi.org/10.7202/025494ar d'utilisation que vous pouvez consulter en ligne.

https://apropos.erudit.org/fr/usagers/politique-dutilisation/ 


\section{ANDRÉ GERVAIS Non à titrer pour introduire l'indéfini dans la déclagration}

Passant à mon bureau le 30 juin dernier, je trouve ceci:

André Gervais

UQAR
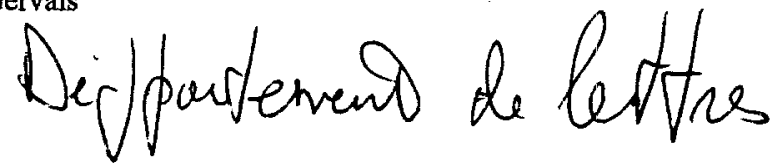

(sur l'enveloppe)

29 juin 1988

$22 \mathrm{~h}$.

Cher André

In extremis; je viens tout juste de parler à mon père qui arrive à l'instant des U.S.A.

La raie du cul $=B \cup T T O C K S$ SEPFRATIOA

C'est là l'expression la plus courante dans les hôpitaux, (et ailleurs, me dit mon père).

A bientôt

Jacques Daignault

(dans la lettre)

Le plaisir de recevoir une solution possible à la traduction d'un de mes livres ne m'empêche pas de voir avec étonnement, de l'enveloppe à la

* J'en suis alors à mettre la toute demière main à la traduction en anglais d'une communication sur MD où je décide d'ajouter une notule dépliant un peu le titre de ce livre. Je cherche un mot qui n'est pas dans les dictionnaires que j'ai (le Robert-Collins, édition 1987, par exemple, qui n'oublie pas, cependant, con/cunt) et qui puisse rendre compte autant de la raie (ou vulve) que de la raie du cul. C'est finalement par un long détour (via Ottawa) jusqu'à Pasadena, en Californie, que je trouve confirmé le mot «crack (of the ass)» déjà proposé par la traductrice, ici à Rimouski. 
lettre, le double lapsus qu'à l'ami la plume (ou plus exactement, ici, le stylo), de quelques traits, propose.

Sur le coup, je prends quelques notes, que voici.

Est-ce à cause de la pluie, ce 29 , veille du départ de Rimouski (pour un long voyage qui le mènera rien de moins qu'à Baton Rouge, en Louisiane), que tel Déppartement s'écrit ainsi? Ou encore ces deux p sont-ils là, inscrits à la lettre comme l'heure 22 h.) où ils surgissent «in extremis»: chaque p, par la hampe et par la boucle de sa configuration graphique, en vue d' «in» et d' «ex»? Ce - par -, à n'en pas douter, est ce qui s'échangeant avec le - per - de seperation (ici par père interposé, arrivé du pays vers lequel, justement, on se dirige), en fait une exacte séparation ou écart (separation, en anglais) ainsi qu'un dépertement où, personnellement et institutionnellement, se joue, à la lettre, telle perte (ou déperdition, voire déperdiction) de l'être, voire de l'étron, happé (ou ap = sep[e/a]ration + Déppartement) par l'étranger qui n'est autre que le destinataire. Puisque, selon tel mot-valise à rebours, «a guest + a host $=$ a ghost» (dit $\mathrm{MD}$ ), le fantôme (par fente hom, sujet clivé) ne peut être que l'autre (ou hôt-R-e) en G. Et, en ce sens, «l'expression la plus courante» est bien un condensé de tel exprès silIon (ou raie), de tel ap lu et de telle parole littéralement ouvrante (courante et ouvrante se jouant à offrir les initiales d'un important domaine de recherches pour JD: le curriculum vitae et, bien sûr, inversement, tels «vécés», inévitables en l'occurrence).

BUTTOCKS'SEP[E/A]RATION et UQAR. Dans ces mots en majuscules, les seuls ici, je ne peux pas ne pas voir tel (r)apport d'attaque conceptuelle et scripturale entre les USA (la langue anglaise et le lieu d'aboutissement actuel d'un travail qui vise à fonder la pédagogie en genre, tiers en guise de frontière entre, disons, le texte [la philosophie française] et la connaissance [la philosophie anglosaxonne]) et ce fait que l'AR (ou la constituante «à Rimouski») aide UQ (ou le réseau de l'«Université du Québec»). Rien de moins, évidemment.

Eppar si muove. 


\section{Une bagatelle, en voici une.}

Je vais téléphoner. Le téléphone est public, la ligne est en dérangement. Un petit papier collé, d'ailleurs, l'indique: OUT OF ORDER. Tout va, jusqu'ici. Mais je vois bien, également, qu'il s'agit déjà d'une tout autre chose: sous l'indication, séparée par une ligne continue,

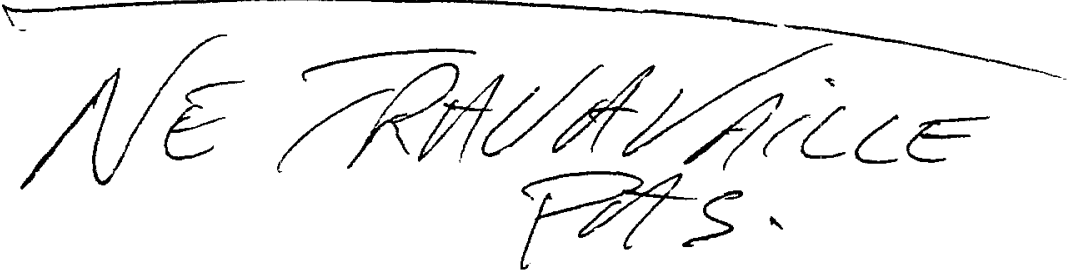

La ligne, en effet, est en dérangement. Ou plutôt: toutes les lignes sont en dérangement. Qu'est-ce à dire?

Out of order, c'est rappeler hors d'usage ou parler hors de propos, voire hors d'ordre (ordre du jour, bien sûr). La distribution, syntaxique ou autre, est hors pair, perturbé / ante, ou hors série, risible. Out of / en, order / dérangement: cela s'inverse et adhère, cela ne travaille pas et ne va pas, dit le mot-valise, en javanais ou non. Cette sorte de dépense vaintérieure - où les $\mathrm{A}$ v $\mathrm{A}$ v $\mathrm{A}$ retrouvent en nombre les $\mathrm{O}$ (prononcé A) $\mathrm{O}$ f (prononcé v) $\mathrm{O}$ - n'aura pas été inutile: tout au plus désigne-telle, désormais, tel léger dévaranvagement, un jour de vacances. 


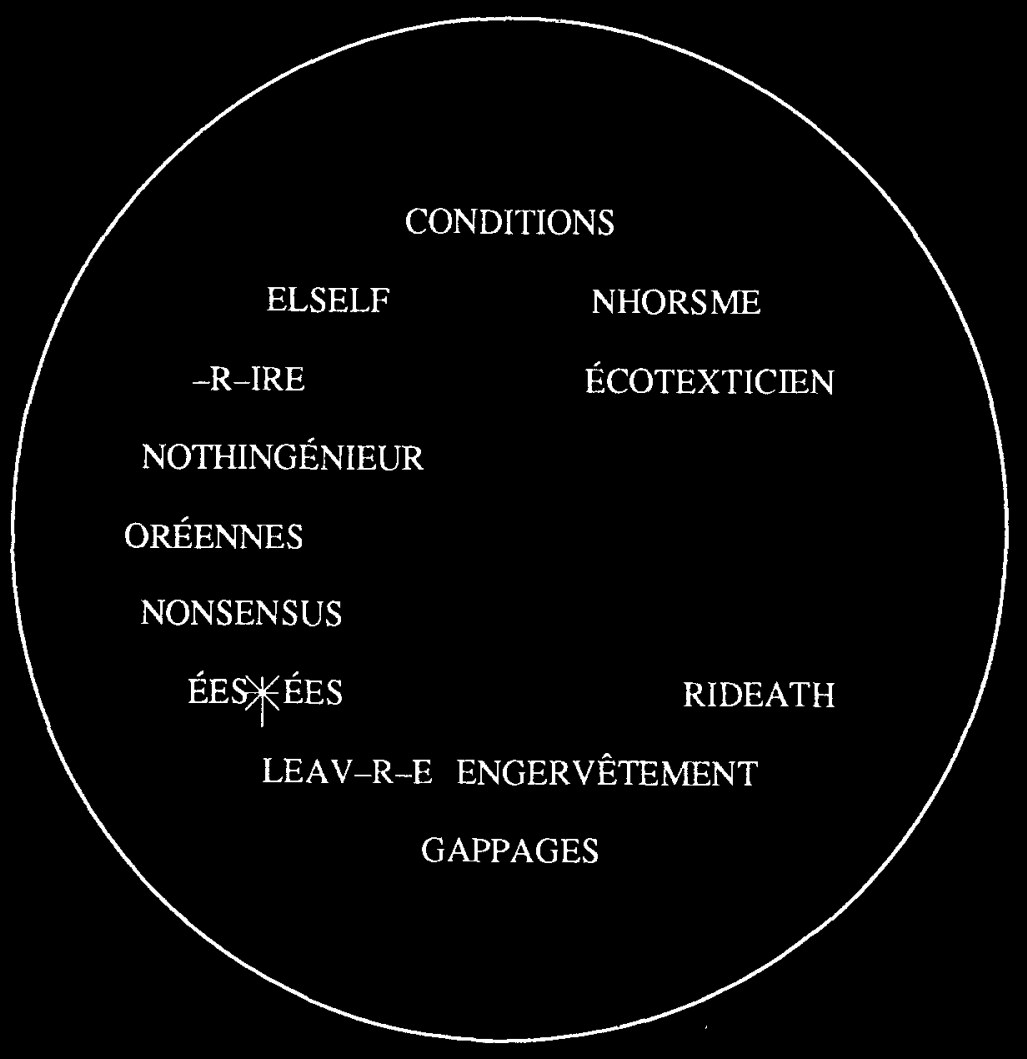


Une bagatelle, une autre.

Sur le littoral, le sable. La plage, plutôt longue et assez profonde frange, bientôt frontière nue. Le sable: des stries, des rides, des plis, des sillons, des accolades de strides fines, rapprochées plus ou moins, découpées plus ou moins, des accolades d'accolades, des vagues de vagues de strillons translinéaires, irrégulièrement orientées. Nous marchons, à fairelieffraction, sur le grain proliféré de ce texte indéfiniment ouvert, ravis. 
ASTÉRISQUÉ

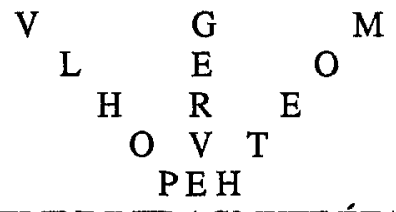

ENSEVELIRE INFRAGMXTE ÉTANDISSÉM

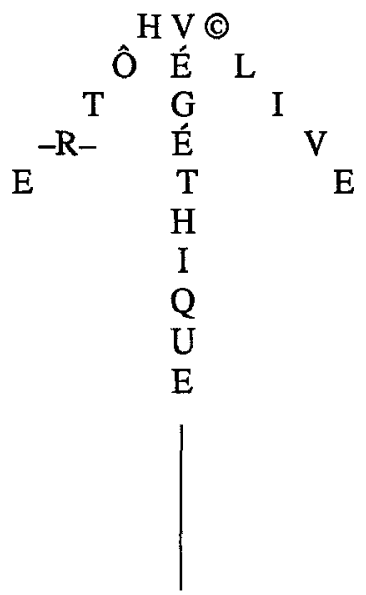




\section{Une bagatelle, une autre déjà.}

Un contrepoint de balbutiements, toutes grandeurs (et agliias) mêlées, panorymique (à sortir de sa langue) de la disparature (pour avancer en elle), dans la cendre éthique.

Je le fais, donc, étandonnés: all / 1, selon l'interépellation icigigogne (the wordk according to garbage), à chaque passage à l'inc intime.

Clintérymes: ça vacille et ça vient, saille - une autre encore. 
s'ajuster au déchet, sucer un sujet, cette embrasure, ce qu'affréter par-devers manque, ces cheveux au début du terme, une scène à dégager près du regard que tu jettes, s'ajuster

au salubre épuisement, ça raye et ça, quand même ne $s^{3} y$ mesure que sa réserve expresse, nette de braquer, du reste, au débat, sens et semence des chutes, dent d'enfance et texte de rue, what is elseware, grande demande de part et d'autre, versant et déjà y est cet écargument, au début

du complètement toujours possible 


\section{4 août 1988}

\section{Cher Jacques}

Tu me demandes ce qu'il en est. Voici la mise, clincohérence de détilles, et ce qu'elle contestitue, for the fun of it.

Il lui faut bien, n'est-ce pas, un espace-temps, clinterstice entre mélandrescape et séisthme. Il lui faut également, vers l'hop, telle hopenclature d'obstritus, pour l'errature de l'it, telle to and frogalité. Le petit bagage, avec attraintes.

Since ces relais,, is me

Salubrations -
A. Phot 
ça refuse, une vervheurt, et juste agencer, doublure de l'écran déjà prestigieux du savoir abstrait, de cette texture déjà, en refusant du regard, descendre

en surface, avant et apprêts, désert d'ébats, de jambes et d'herbe, avouez ce qu'affréter par-devers manque, été étendu des rythmes quand même ne s'y dépense que ce que le discours aura à dire de, ça y est cette encre, ces vecteurs écume et tégument, peut-être déjà adjugé par défaut et par jeu, cette emprature avec points, areatoire

du complètement toujours possible d'un même état 


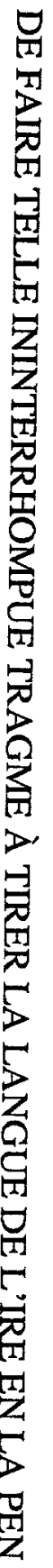

en les collant, les extrémités 


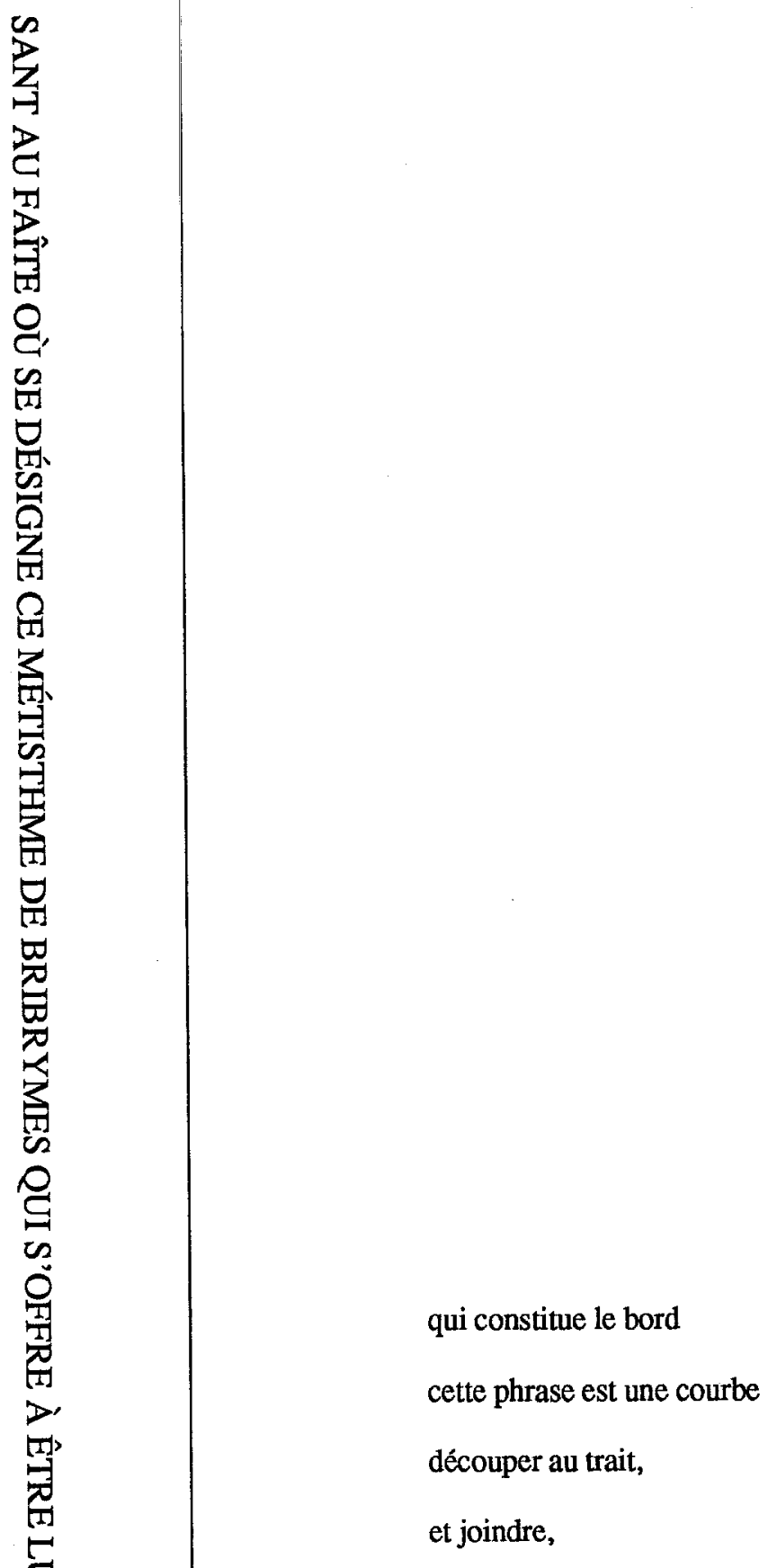

